\title{
Real-space mapping of the chiral near-field distributions in spiral antennas and planar metasurfaces
}

\section{- Supplementary information -}

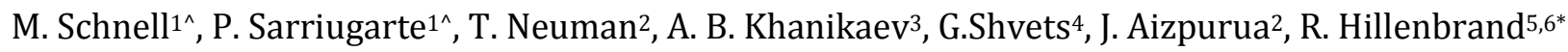

${ }^{1}$ CIC nanoGUNE Consolider, 20018 Donostia - San Sebastián, Spain

${ }^{2}$ Centro de Fisica de Materiales CSIC-UPV/EHU and Donostia International Physics Center DIPC, 20018 Donostia - San Sebastián, Spain

${ }^{3}$ Department of Physics, Queens College and Graduate Center of The City University of New York, New York 11367, USA

${ }^{4}$ Department of Physics, The University of Texas at Austin, Austin, Texas 78712, USA

${ }^{5}$ CIC nanoGUNE Consolider and EHU/UPV, 20018 Donostia - San Sebastián, Spain

${ }^{6}$ IKERBASQUE, Basque Foundation for Science, 48011 Bilbao, Spain

^equally contributing authors

* corresponding author:

r.hillenbrand@nanogune.eu 
(a)
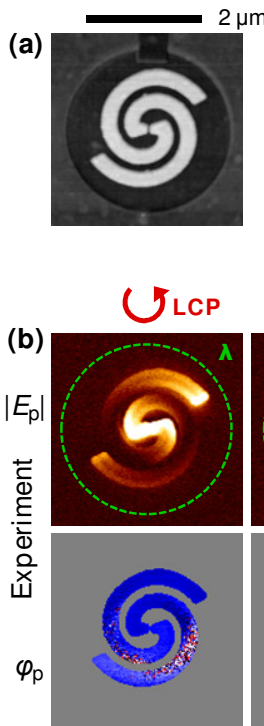

(c)
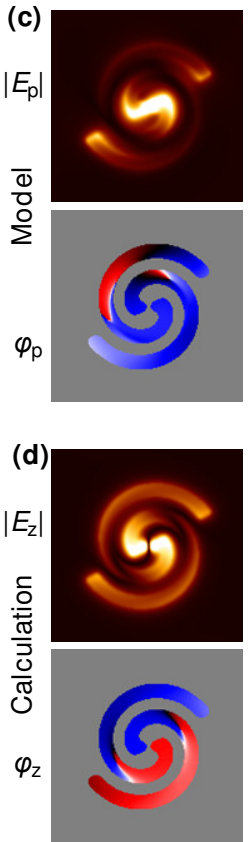

(e)
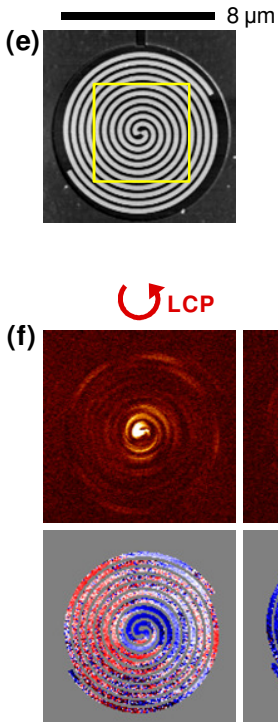

(g)

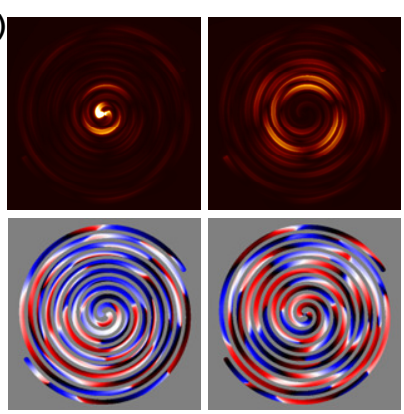

(h)

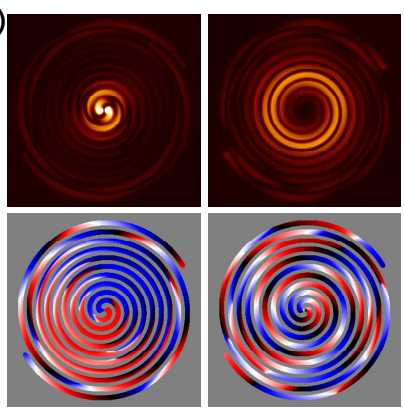

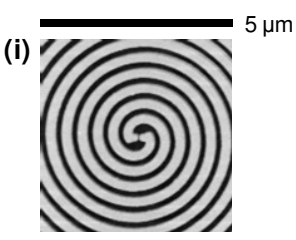
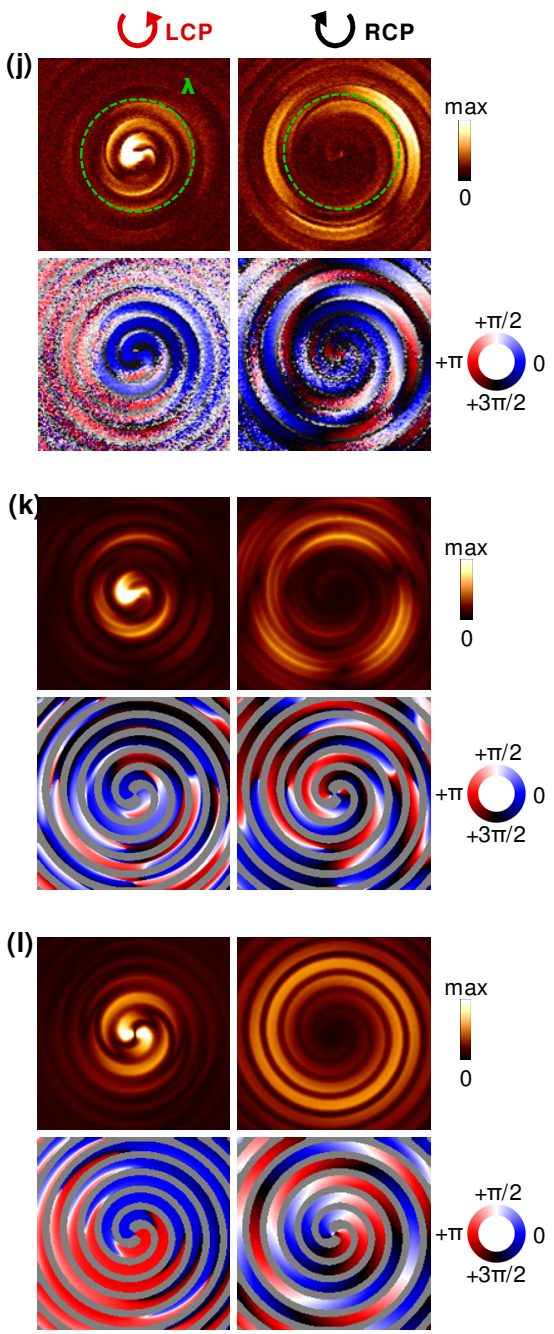

Figure S1: Full data set to Fig. 2 in the main text, showing the chiral near-field distributions of planar Archimedean spiral antennas operating at infrared frequencies. (a) Topography image of a 1-turn spiral antenna. (b) Experimental s-SNOM images of the antenna in (a). Shown is the near-field amplitude $\left|E_{\mathrm{p}}\right|$ and phase $\varphi_{\mathrm{p}}$ for left-handed (LCP) and right-handed circular polarized (RCP) illumination. The amplitude color scale is saturated to show the antenna mode more clearly. (c) Modeled s-SNOM images, $\left|E_{\mathrm{p}}\right|$ and phase $\varphi_{\mathrm{p}}$. (d) Numerically calculated vertical electric field component, $\left|E_{\mathrm{z}}\right|$ and $\varphi_{\mathrm{z}}$. (e) Topography image of a 5-turn spiral antenna, overview. (fh) Experimental, modeled s-SNOM images and numerical calculations of the antenna in (e). (i) Higher-resolution rescan of the area marked by the yellow box in (e). (j-l) Experimental, modeled sSNOM images and numerical calculations corresponding to (i). 
(a)

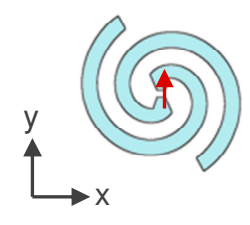

(c)

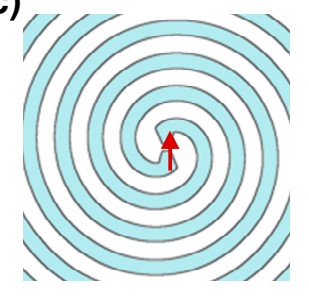

(b) $\varphi=90^{\circ}$

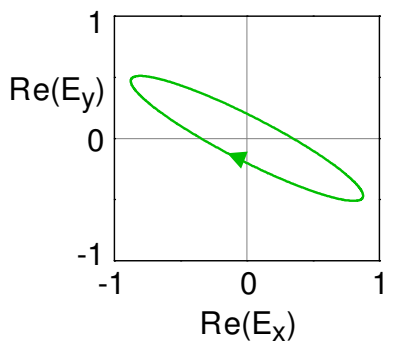

(d) $\varphi=90^{\circ}$

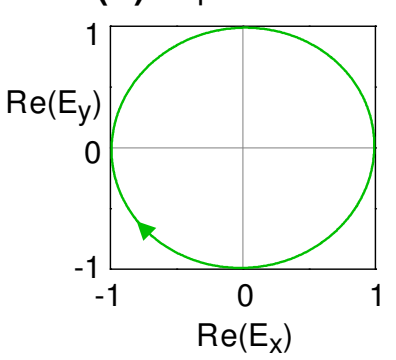

Figure S2: Calculated radiation characteristics of our infrared 1-turn and 5-turn Archimedean spiral antennas shown in Fig. 2 in the main text. The data are obtained by FDTD simulations assuming a wavelength of $\lambda=9.3 \mu \mathrm{m}$. (a) Sketch of the 1-turn spiral antenna excited by an electric dipole placed inside the antenna gap. (b) Polarization state of the radiated light in $+\mathrm{z}-$ direction, showing an elliptical polarization state of axial ratio 5.5:1. (c) Sketch of the 5-turn spiral antenna excited by an electric dipole placed inside the antenna gap. (d) Polarization state of the radiated light in $+z$-direction, showing a circular polarization state (axial ratio is 1.02:1). 
(a)

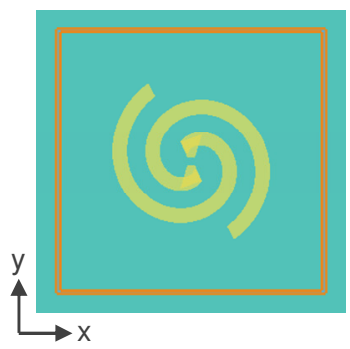

(c)

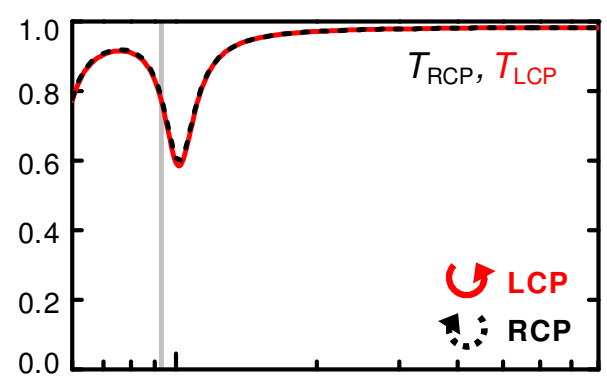

(e)

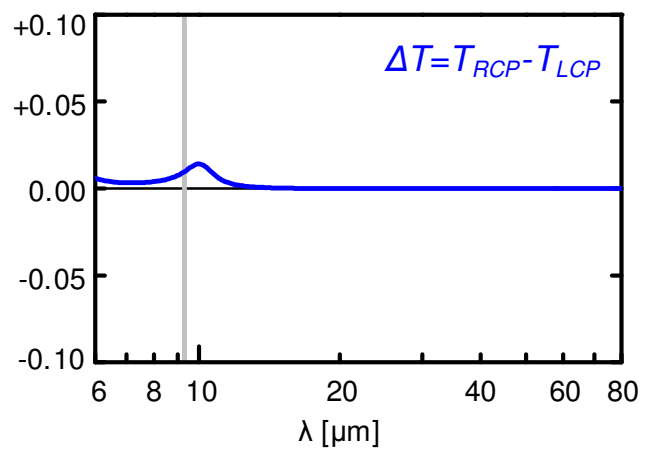

(b)

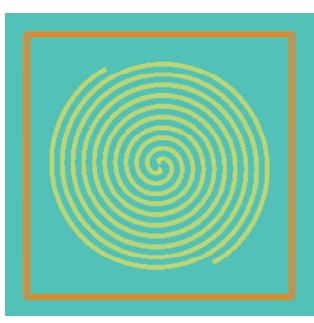

(d)

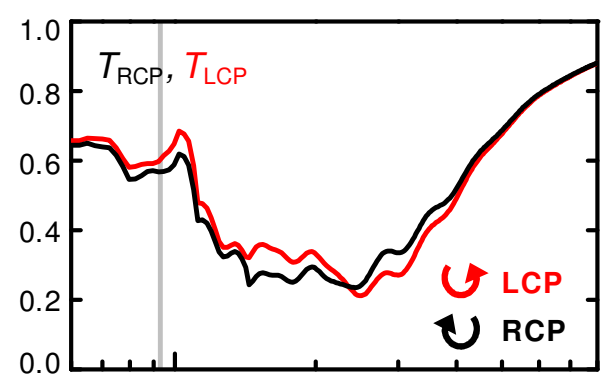

(f)

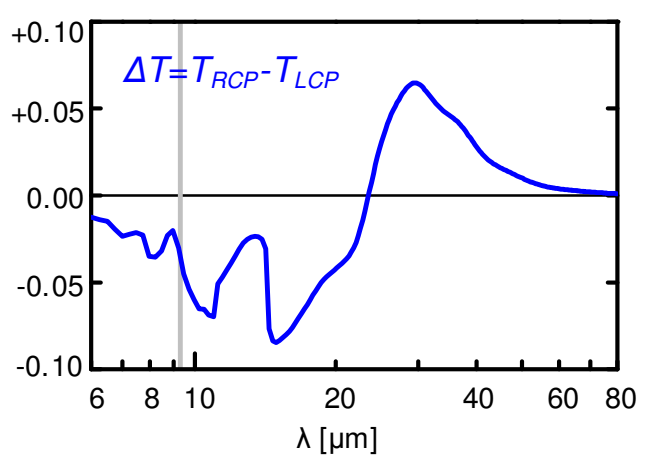

Figure S3: Circular dichroism (CD) and CD resonances in single-layer metasurfaces consisting of infrared spiral antennas. The data are obtained by FDTD simulations. (a,b) Unit cell of the metasurface showing a 1-turn and 5-turn spiral antenna (yellow color) on top of a dielectric substrate (turquoise color, $\mathrm{n}=1.3$ ) and placed in a box with periodic boundaries in $\mathrm{x}$ and $\mathrm{y}$ (orange color) measuring $4 \mu \mathrm{m}(\mathrm{a})$ and $11 \mu \mathrm{m}$ (b) in width. The structures are illuminated from below in $+\mathrm{z}$ direction and the transmitted fields are recorded above the structure. (c,d) Calculated transmission spectra for LCP and RCP illumination, $T_{\mathrm{LCP}}$ and $T_{\mathrm{RCP}}$. (e,f) CD spectra calculated as difference in transmission, $\Delta T=T_{\mathrm{RCP}}-T_{\mathrm{LCP}}$. The vertical grey line marks the excitation wavelength of $9.3 \mu \mathrm{m}$ as used in the experiments presented in the main text. The 1-turn antenna shows a fundamental dipolar resonance near $10 \mu \mathrm{m}$ wavelength (c). The difference between LCP and RCP is small, yielding CD resonance of +0.014 (e). In comparison, the 5-turn antenna shows complex transmission spectra, which are noticeably different for LCP and RCP. The calculations predict a CD resonance around $32 \mu \mathrm{m}$ wavelength (peaking with +0.064 ) and two further CD resonances around $10 \mu \mathrm{m}$ and $15 \mu \mathrm{m}$ wavelength, peaking with -0.068 and -0.084 , respectively (f). Note that the 1 -turn antenna already shows small CD that is more pronounced than the negligible CD of the rosettes shown in Fig. 4a of the main text, which we attribute to the even shorter arm length of the rosettes (being comparable to a quarter-turn spiral antenna) in comparison to the 1-turn spiral antenna. Furthermore, while the near-field patterns for the rosettes are identical for LCP and RCP, quantitative differences can be observed for the 1-turn spiral antenna (Fig. 2a) explaining the small 
CD observed in panels (c,e). We also note that in the examples of $(a, b)$ the packaging density of the 1-turn antenna is about 7.5-times larger than that of the 5-turn antenna. Assuming the same packaging density (i.e. assuming an $11 \mu \mathrm{m}$ wide unit cell), we obtain a CD of 0.0015 for the 1-turn spiral antenna which corresponds to only $2 \%$ of the maximum CD observed with the 5-turn spiral antenna. 